\title{
ANÁLISE QUANTITATIVA DE DIFERENTES CERÂMICAS ODONTOLÓGICAS SUBMETIDAS AOS PROCEDIMENTOS CLÍNICOS DE ACABAMENTO E POLIMENTO
}

\author{
Bethânia da Silva Santos'; Adriana Castro Vieira Andrade ${ }^{2}$; Alex Vieira Correia ${ }^{3}$ e \\ Maria Célia Nunes Pedreira ${ }^{4}$
}

1. Bolsista PROBIC UEFS, Graduanda em Odontologia, Universidade Estadual de Feira de Santana, e-mail: santos.bethania@yahoo.com.br

2. Orientador, Departamento de Saúde, Universidade Estadual de Feira de Santana, e-mail: dria-castro@ hotmail.com

3. Coordenador do projeto Análise quantitativa e qualitativa da superfície de diferentes tipos de cerâmicas odontológicas submetidas aos procedimentos clínicos de acabamento e polimento, Departamento de Saúde, Universidade Estadual de Feira de Santana, e-mail: dr.alexvieira@hotmail.com

\begin{abstract}
4. Participante do projeto Análise quantitativa e qualitativa da superfície de diferentes tipos de cerâmicas odontológicas submetidas aos procedimentos clínicos de acabamento e polimento, Graduanda em Odontologia, Universidade Estadual de Feira de Santana, e-mail: mariaceliapedreira@gmail.com
\end{abstract}

PALAVRAS-CHAVE: Cerâmicas Odontológicas; Acabamento; Polimento Superficial.

\section{INTRODUÇÃO}

As cerâmicas odontológicas são materiais com excelentes propriedades estéticas, boa biocompatibilidade aos tecidos orais, grande longevidade e boa lisura superficial, o que as tornam uma importante alternativa para a substituição de dentes perdidos ou danificados (Asai, et al. 2010; Dalkiz, et al.2009; Nakamura, et al. 2010). Entretanto, a utilização deste material requer, com frequência, a realização de ajustes clínicos que contribuem para a remoção da camada mais superficial da cerâmica (Sarikaya, et al. 2010).

A remoção dessa camada mais superficial acarreta no aumento da aspereza da cerâmica. A rugosidade criada pode causar problemas clínicos importantes como; o desgaste do dente antagonista (Monasky, et al. 1971), retenção de biofilme bacteriano (Queiroz, et al. 2013), inflamação dos tecidos periodontais, alteração de cor (Haywood, et al. 1989) insatisfação estética (Haywood, et al. 1988) e menor resistência às trincas (Sarikaya, et al. 2010; Flury, et al. 2010). Como alternativa a esses problemas, estudos apontam sobre a necessidade da realização de um reglazeamento (Newitter, et al. 1982) ou acabamento e polimento nessas restaurações (Haroon, et al. 2016).

Em vista do exposto e da controvérsia sobre o tema, este estudo teve como objetivo avaliar quantitativamente a rugosidade superficial de duas diferentes cerâmicas odontológicas, submetidas a diferentes técnicas de acabamento com mesmo polimento.

\section{MATERIAIS E MÉTODOS}

Pesquisa in vitro com 80 corpos de prova de cerâmica. Primeiro grupo composto por 40 amostras de cerâmica feldspática e o segundo com 40 amostras de cerâmica de dissilicato de lítio. Cada Grupo foi subdividido em 4 partes contendo cada um, 10 amostras, Cerâmicas Feldispáticas (G1,G2,G3 e G4) e Cerâmicas de Dissilicato de lítio (G5,G6,G7 e G8). O G1 e o G5 constituíram os grupos controle, permanecendo com suas superfícies glazeadas. Os demais sofreram desgaste simulando o ajuste oclusal e receberam acabamentos distintos e polimentos iguais. 


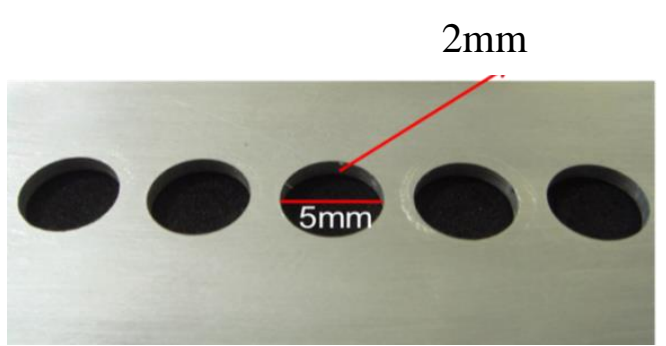

Figura 1- Matriz metálica com orifícios

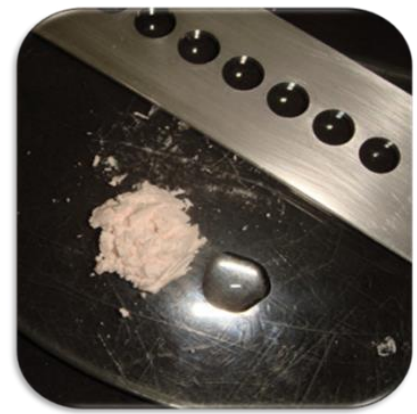

Figura 2- Pó cerâmico e água destilada

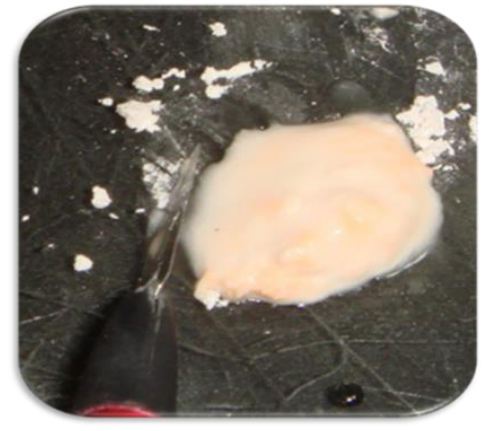

Figura 3- Pó cerâmico sendo incorporado à água destilada e manipulado

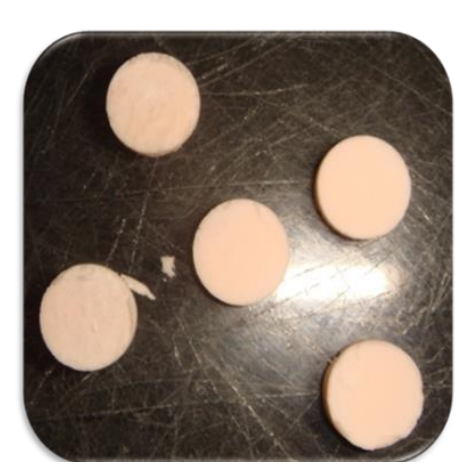

Figura 6- Corpos de prova após remoção dos orifícios

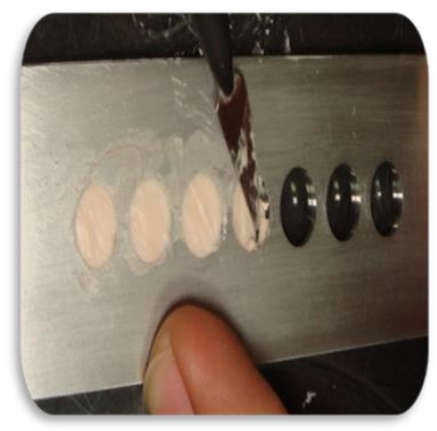

Figura 4- Cerâmica sendo levada aos orifícios da matriz
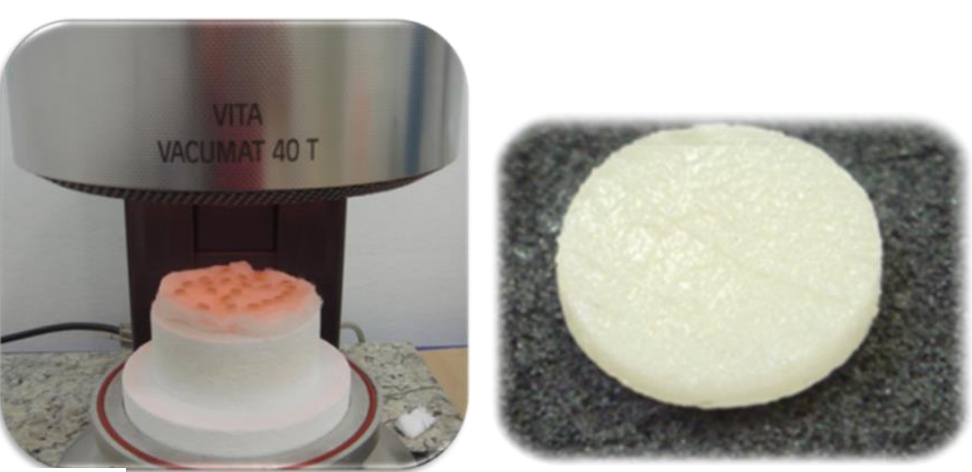

Figura 5- Orifícios

completamente preenchidos

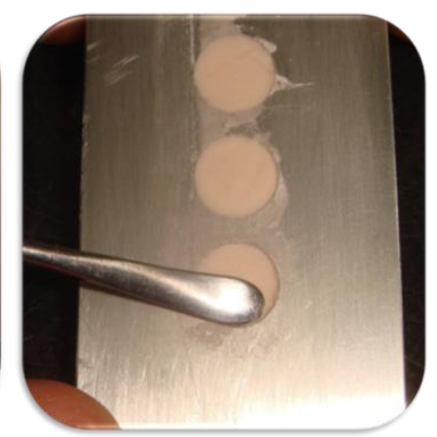

Figura 7- Corpos passando pel Figura 8- Corpo de prova após a processo de queima queima

\section{RESULTADOS}

A tabela 1 apresenta os valores da rugosidade superficial média ( $\mathrm{Ra}$ ) dos grupos testados. De acordo com os resultados encontrados, para a cerâmica feldspática, o grupo controle (G1) apresentou o menor valor de rugosidade superficial com diferença estatisticamente significante $(\mathrm{p}<0,05)$ em relação aos grupos tratados com os diferentes sistemas de acabamento e polimento (G2, G3 e G4), sendo que estes não apresentaram diferenças entre si $(p>0,05)$.

Já para a cerâmica E-max, reforçada por dissilicato de lítio, o grupo controle (G5) também apresentou diferença significativa $(\mathrm{p}<0,05)$ quando comparado aos grupos tratados com as borrachas abrasivas Astropol (G6) e Edenta (G7). Porém, o grupo tratado com o 
sistema Sof-Lex Pop-on (G8) não apresentou diferença significativa com o grupo controle (G5). Em relação aos diferentes sistemas de acabamento e polimento utilizados, apenas o grupo tratado com as borrachas Edenta (G7) apresentou diferença significativa com o grupo tratado com o sistema Sof-Lex (G8). G6 e G7 não apresentaram diferenças estatísticas entre si, assim como G6 e G8.

Da mesma forma, quando os grupos das duas cerâmicas, correspondentes aos mesmos tratamentos, são comparados entre si, não foram encontradas diferenças estatísticas significantes $(\mathrm{p}<0,05)$.

Tabela 1- Rugosidade superficial média ( $\mathrm{Ra}$ ) dos grupos experimentais, em $\mu \mathrm{m}$, apresentadas como média \pm desvio padrão $(\mathrm{dp})$

\begin{tabular}{ccc}
\hline GRUPO & MÉDIA (Ra) & $\begin{array}{c}\text { DESVIO } \\
\text { PADRÃO (dp) }\end{array}$ \\
\hline G1 & 0.70 & 0.07 \\
\hline G3 & 1.14 & 0.08 \\
\hline G4 & 1.13 & 0.06 \\
\hline G5 & 1.15 & 0.09 \\
\hline G6 & 0.87 & 0.22 \\
\hline G7 & 1.11 & 0.27 \\
\hline G8 & 1.39 & 0.27 \\
\hline
\end{tabular}

\section{DISCUSSÃO}

De acordo com os resultados apresentados nesta pesquisa, observou-se que todos os métodos de acabamento e polimento testados nas cerâmicas feldspáticas obtiveram uma eficácia semelhante, não havendo entre elas diferenças estatísticas significantes. Além disso, neste grupo, foi possível perceber que nenhum método utilizado proporcionou uma lisura superficial semelhante ou superior à superfície glazeada. Entretanto, ao avaliar o grupo composto pela cerâmica de Dissilicato de lítio, as amostras glazeadas obtiveram lisuras semelhantes às encontradas nas tratadas com discos soflex.

\section{CONCLUSÃO}

As cerâmicas feldspática e reforçada por dissilicato de lítio, quando glazeadas, apresentaram valores similares de rugosidade superficial. Os sistemas de acabamento e polimento utilizados para cerâmica feldspática não se mostraram superiores à aplicação do glaze. A cerâmica reforçada por dissilicato de lítio obteve melhores resultados de rugosidade superficial quando tratada com o glaze e com o sistema Sof-Lex. 


\section{REFERÊNCIAS}

1 ASAI T, KAZAMA R, FUKUSHIMA M, OKIJI T. 2010. Effect of overglazedandpolished surface finishes on the compressive fracture strength of machinable ceramic materials. Dent Mater J;29:661-667.

2 DALKIZ M, SIPAHI C, BEYDEMIR B. 2009. Effects of six surface treatment methods on the surface roughness of a low-fusing and an ultra low fusing feldspathic ceramic material. $\mathrm{J}$ Prosthodont; 18:217-222.

3 NAKAMURA Y, SATO H, OHTSUKA M, HOJO S. 2010. Polishing of dental porcelain by polycrystalline diamond. Biomed Mater Eng;20:283-293.

4 SARIKAYA I, GU* LER AU. 2010. Effects of different polishing techniques on the surface roughness of dental porcelains. J Appl Oral Sci;18:10-6.

5 MONASKY GE, TAYLOR DF. 1971. Studies on the wear of porcelain, enamel, and gold.JprosthetDent;25:299-306.

6 QUEIROZ JR, FISSMER SF, KOGA-ITO CY, SALVIA ACRD, MASSI M, SOBRINHO AS, et al. 2013. Effect of diamond-like carbon thin film coated acrylic resin on Candida albicans biofilm formation. J Prosthodont;10:120-9.

7 HAYWOOD VB, HEYMANN HO, SCURRIA MS. 1989. Effects of water, speed, and experimental instrumentation on finishing and polishing porcelain intra-orally. Dent Mater;5:185-8.

8 HAYWOOD VB, HEYMANN HO, KUSY RP, WHITLEY JQ, ANDREAUS SB. 1988. Polishing porcelain veneers: an SEM and specular reflectance analysis. Dent Mater;4:116-21. 9 FLURY S, LUSSI A, ZIMMERLI B. 2010. Performance of different polishing techniques for direct CAD/CAM ceramic restorations. Oper Dent;35:470-81.

10 NEWITTER DA, SCHLISSEL ER, WOLFF MS. 1982. An evaluation of adjustment and postadjustment finishing techniques on the surface of porcelain-bonded-to-metal crowns. $\mathrm{J}$ Prosthet Dent;48:388-95.

11 HAROON R, ZEESHAN S, SYED M, MURTAZARK, SAMEER Q, MUHAMMAD ZU. 2016. Advancements in all-ceramics for dental restorations and their effect on the wear of opposing dentition.European Journal of Dentistry, Vol 10 / Issue 4 / Oct-Dec. 